\title{
Multi-layer Scaffolds of Poly(caprolactone), Poly(glycerol sebacate) and Bioactive Glasses Manufactured by Combined 3D Printing and Electrospinning
}

\author{
Adja B. R. Touré ${ }^{1,2, *}$, Elisa Mele ${ }^{1}$ (D) and Jamieson K. Christie ${ }^{1}$ \\ 1 Department of Materials, Loughborough University, Loughborough LE11 3TU, UK; \\ e.mele2@lboro.ac.uk (E.M.); j.k.christie@lboro.ac.uk (J.K.C.) \\ 2 Centre for Additive Manufacturing, Faculty of Engineering, Jubilee Campus, Nottingham University, \\ Nottingham NG7 2RD, UK \\ * Correspondence: adja.toure@nottingham.ac.uk
}

Received: 29 February 2020; Accepted: 25 March 2020; Published: 28 March 2020

\begin{abstract}
Three-dimensional (3D) printing has been combined with electrospinning to manufacture multi-layered polymer/glass scaffolds that possess multi-scale porosity, are mechanically robust, release bioactive compounds, degrade at a controlled rate and are biocompatible. Fibrous mats of poly (caprolactone) (PCL) and poly (glycerol sebacate) (PGS) have been directly electrospun on one side of 3D-printed grids of PCL-PGS blends containing bioactive glasses (BGs). The excellent adhesion between layers has resulted in composite scaffolds with a Young's modulus of $240-310 \mathrm{MPa}$, higher than that of 3D-printed grids (125-280 MPa, without the electrospun layer). The scaffolds degraded in vitro by releasing PGS and BGs, reaching a weight loss of $\sim 14 \%$ after 56 days of incubation. Although the hydrolysis of PGS resulted in the acidification of the buffer medium (to a $\mathrm{pH}$ of 5.3-5.4), the release of alkaline ions from the BGs balanced that out and brought the $\mathrm{pH}$ back to 6.0. Cytotoxicity tests performed on fibroblasts showed that the PCL-PGS-BGs constructs were biocompatible, with cell viability of above $125 \%$ at day 2 . This study demonstrates the fabrication of systems with engineered properties by the synergy of diverse technologies and materials (organic and inorganic) for potential applications in tendon and ligament tissue engineering.
\end{abstract}

Keywords: composite biomaterials; porous scaffolds; degradation

\section{Introduction}

In the field of tissue engineering, combinations of different materials and fabrication approaches are investigated to manufacture scaffolds that are able to satisfy more than one of the following requirements simultaneously [1-3]: be biocompatible; offer a biomimetic interface and multiscale porosity to promote cell attachment, migration and proliferation; mimic the mechanical response of the native tissue; and degrade, if required, at a controlled rate to support tissue regeneration.

Recently, blends of poly(caprolactone) (PCL), a slow-degrading polyester, and poly(glycerol sebacate) (PGS), a fast-degrading polyester, have been processed by either electrospinning or 3D printing to create porous and biodegradable scaffolds with controlled mechanical properties for heart valve replacement [4-6], cardiac patches [7-9] and corneal tissue repair [10]. In one study, the degradation of PGS-PCL electrospun scaffolds (with a 2:1 PGS:PCL weight ratio) has been investigated in accelerated conditions in an alkaline medium $(0.1 \mathrm{mM} \mathrm{NaOH})$ and in vitro using valvular interstitial cells (VICs) [5]. Blending PGS with PCL led to a $16 \%$ mass loss of the scaffolds in 7 days, while fibrous mats of just PCL showed a $6 \%$ mass loss at the same timepoint. The PGS-PCL 
scaffolds stimulated VICs to secrete extracellular matrix (ECM) proteins (collagen I, laminin and fibronectin), which contributed to the mechanical properties of the cell-seeded scaffolds. A Young's modulus of 9.3 MPa was recorded for PGS-PCL scaffolds seeded with VICs after 3 weeks of culture (7.8 MPa at day 0); while acellular scaffolds showed a two-fold decrease in elastic modulus under the same conditions. The enhanced secretion of ECM proteins and the control achieved over degradation and mechanical properties of the scaffolds indicate that the PGS-PCL electrospun mats can be used for heart valve tissue engineering.

In another work, PGS-PCL electrospun fibres have been functionalised with vascular endothelial growth factors (VEGFs) and tested as cardiac patches [7]. The PGS-PCL fibres (with a 2:1 PGS:PCL weight ratio) were characterised by a Young's modulus of $8 \mathrm{MPa}$, which decreased to $1.4 \mathrm{MPa}$ after 28 days of degradation in phosphate-buffered saline solution (PBS) at $37^{\circ} \mathrm{C}$, due to the breakdown of the ester bond and hydrolysis of PGS (resulting in 57\% weight loss). VEGF was immobilised onto the scaffolds by 1-ethyl-3-(3-dimethylaminopropyl) carbodimide hydrochloride)/N-hydroxysuccinimide (EDC-NHS) chemistry and its release kinetics were investigated in water at $37^{\circ} \mathrm{C}$. The scaffolds showed a VEGF release of around $90 \%$ after 30 days and almost complete release after 50 days, possibly caused by the surface hydrolytic degradation of the fibres. The VEGF-functionalised mats promoted the adhesion and growth of mouse myoblast cell line C2C12 and rat cardiac progenitor cells (rCPCs), showing potential as tissue-engineered cardiac patches.

Aiming at improving cardiac remodelling after myocardial infarction, 3D printing of mixtures of PGS-PCL (with a PGS:PCL 9:1 weight ratio) and sacrificial sodium chloride particles has been performed [9]. Due to the high PGS concentration and 60\% porosity, the scaffolds exhibited a Young's modulus of $0.7 \mathrm{MPa}$ and fast degradation ( $90 \%$ in $12 \mathrm{~h}$ ) in highly concentrated PBS solution of lipase from Thermomyces lanuginosus. The scaffolds were implanted in adult male rats 2 days after left coronary ligation. Four weeks after implantation, the 3D-printed PGS-PCL scaffolds induced improvements in cardiac functions, decreased infarct size, and increased the thickness of the left ventricle wall and tissue remodelling.

TO date, electrospinning and 3D printing of PCL-PGS blends have been demonstrated separately to obtain scaffolds with controlled properties. Here, we investigate, for the first time, PCL-PGS constructs consisting of electrospun mats deposited onto 3D-printed scaffolds. Bioactive glasses have been incorporated into the 3D printed polymer matrix to achieve better control over mechanical properties, degradation profile and biocompatibility. The composite PCL-PGS scaffolds formed of electrospun and 3D printed layers were characterised by a Young's modulus in the range of 250-310 $\mathrm{MPa}$, and experienced a weight loss of 10-15\% after 56 days of incubation in a buffer solution. Hybrid fabrication strategies, like the combination of electrospinning and 3D printing, are discussed in the literature to introduce biomimetic features and multiscale porosity within additive manufactured scaffolds [11]. Here, the 3D printed layer provided mechanical support, maintained the shape integrity of the composite scaffolds and allowed the release of bioactive compounds. The electrospun layer was instead used to further engineer mechanical properties and porosity, and make biological inducible structures available to cells.

\section{Materials and Methods}

\subsection{Synthesis of PGS}

PGS was synthesised following the procedure reported by Wang and collaborators [12]. A round-bottomed flask equipped with a $\mathrm{N}_{2}$ bubbler and a Dean-Stark trap was filled with an equimolar mixture of anhydrous glycerol (Sigma Aldrich, purity 99\%, Gillingham, UK) and recrystallized sebacic acid (Sigma Aldrich, purity 99\%). Bubbling $\mathrm{N}_{2}$ was passed through the mixture for 10 min before the compounds were heated up to $120^{\circ} \mathrm{C}$ for $24 \mathrm{~h}$. The pressure was then reduced from 1 torr to $40 \mathrm{mtorr}$ over $5 \mathrm{~h}$. The reaction mixture was kept at 40 mtorr and $120^{\circ} \mathrm{C}$ for $48 \mathrm{~h}$ to crosslink the prepolymer. 


\subsection{Fabrication of $3 D$ Printed Scaffolds}

An extrusion printer 3D-Bioplotter Developer Series (EnvisionTec, Gladbeck, Germany) was used to produce three types of 3D printed scaffolds: PCL-PGS (hereafter referred to as 3D PCL-PGS), PCL-PGS containing $5 \mathrm{wt} \%$ of bioactive glasses (3D PCL-PGS-5BGs), and PCL-PGS containing $10 \mathrm{wt} \%$ of bioactive glasses (3D PCL-PGS-10BGs). PCL with a molecular weight of $\sim 80,000 \mathrm{Da}$ (Sigma Aldrich) was used. The bioactive glass (BG) microspheres (45S5, XL Tech science, Richland, WA, USA) were formed of $45.0 \mathrm{wt} \% \mathrm{SiO}_{2}, 24.5 \mathrm{wt} \% \mathrm{CaO}, 24.5 \mathrm{wt} \% \mathrm{Na}_{2} \mathrm{O}$ and $6 \mathrm{wt} \% \mathrm{P}_{2} \mathrm{O}_{5}$. The $\mathrm{BGs}(0.25$ and $0.50 \mathrm{~g}$ for 3D PCL-PGS-5BGs and 3D PCL-PGS-10BGs, respectively) were firstly dispersed in $50 \mu \mathrm{L}$ of dimethyl sulfoxide (DMSO, Sigma Aldrich) and $10 \mathrm{~mL}$ of acetone (Sigma Aldrich). The dispersion was stirred for $1 \mathrm{~h}$ using a magnetic stirrer under vigorous agitation, before adding $2.5 \mathrm{~g}$ of PGS. Following the complete dissolution of PGS at $40{ }^{\circ} \mathrm{C}, 2.5 \mathrm{~g}$ of PCL were added, and the dispersion was left stirring at $40{ }^{\circ} \mathrm{C}$ for $12 \mathrm{~h}$.

Barrels containing the solutions were individually loaded into the Bioplotter and dispensed through smooth-flow tapered tips ( $340 \mu \mathrm{m}$ internal diameter) from $12.7 \mathrm{~mm}$ and extruded on to an aluminium build platform coated with an aluminium foil. Pressure and speed time for pre-flow and post-flow were adjusted and optimised to obtain the desirable inner structure for each composition. The printing process was conducted at $37^{\circ} \mathrm{C}$. The samples (square grids, area $4 \times 4 \mathrm{~cm}^{2}$ ) consisted of layers formed of parallel strands placed at a $1.45 \mathrm{~mm}$ distance from one to another. Each layer was deposited perpendicular to the previous one, in order to form a grid.

For the fabrication of multi-layer constructs, the 3D-printed samples were left to dry on the aluminium foil and then used as a collector for the electrospinning process. PCL and PGS were mixed at 1:1 weight ratio and dissolved in a 7:3 (weight ratio) mixture of dichloromethane (Sigma Aldrich) and methanol (Sigma Aldrich) to obtain a $14 \mathrm{wt} \%$ polymer solution. A plastic syringe with a 21G needle $(0.80 \mathrm{~mm}$ in diameter and $120 \mathrm{~mm}$ in length) was filled with the polymer solution, prepared, and connected to a syringe pump (New Era Pump System, NE-300, New York, NY, USA), working at a flow rate of $0.8 \mathrm{ml} / \mathrm{h}$. The needle was connected to the positive electrode of a high-voltage power supply (S1500032-0, Linari Engineering s.r.l., Pisa PI, Itlay), generating a voltage of $8 \mathrm{kV}$, while the ground electrode was fixed to an aluminium collector (air gap distance of $15 \mathrm{~cm}$ ). All experiments were conducted in normal environmental conditions. The PCL-PGS solution was electrospun only on one surface of the 3D-printed scaffolds. After production, the samples were stored at room temperature.

\subsection{Characterisation Procedures}

\subsubsection{Nuclear Magnetic Resonance}

${ }^{1} \mathrm{H}$ Nuclear Magnetic Resonance (NMR) spectra of PGS were recorded in commercial deuterated solvent on a JEOL ECS-400 spectrometer (JEOL (UK) Ltd., Herts, UK; ${ }^{1} \mathrm{H}$ at $399.782 \mathrm{MHz},{ }^{13} \mathrm{C}$ at $100.525 \mathrm{MHz}$ ), a Bruker Advance Ultra-Shield 400 spectrometer (Bruker AXS Ltd., Coventry, UK; ${ }^{1} \mathrm{H}$ at $400.134 \mathrm{MHz},{ }^{13} \mathrm{C}$ at $100.624 \mathrm{MHz}$ ) at $293 \mathrm{~K}$. Chemical shifts were expressed as $\delta$ in parts per million $(\mathrm{ppm})$ and were adjusted to the chemical shift of the residual NMR solvent resonance peak $\left(\mathrm{CDCl}_{3},{ }^{1} \mathrm{H}\right.$ : $\delta=7.26 \mathrm{ppm})$.

\subsubsection{Fourier Transform Infrared Spectroscopy}

The infrared spectra of PGS were recorded on the neat compound using a Fourier Transform Infrared (FTIR) Spectrophotometer Shimadzu FTIR-8400S (Shimadzu Europa Gmbh, Duisburg, Germany), equipped with an Attenuated Total Reflection (ATR) diamond and irradiating between 7800 and $350 \mathrm{~cm}^{-1}$, at 64 scans and a resolution of $4 \mathrm{~cm}^{-1}$. The data were recorded through the software IR solution. 


\subsubsection{X-ray Diffraction}

The BG microspheres were dispersed in acetone and the suspension was placed onto a silicon substrate to produce a thin smear. The sample was analysed by a Bruker D8 Advance diffractometer (Bruker AXS Ltd.) in reflection geometry, $\mathrm{Cu} \mathrm{K} \alpha$ radiation. The data were collected over the $2 \theta$ range from $5^{\circ}$ to $60^{\circ}$ with a step size of $0.014^{\circ}$ and a count time of $5.5 \mathrm{~s}$ per step.

\subsubsection{Scanning Electron Microscopy}

The morphology of BG microspheres, electrospun fibres and 3D printed scaffolds was investigated by Field Emission Gun Scanning Electron Microscopy (FEGSEM, LEO 1530VP, LEO Elektronenmikroskopie GmbH, Oberkochen, Germany). Prior to observation, the samples were stuck on aluminium stubs by carbon adhesive tapes and then coated using a palladium/gold sputter coater for $90 \mathrm{~s}$ (Emitech SC7640 Sputter Coater, Polaron, Laughton, UK) to produce a conductive surface.

\subsubsection{Uniaxial Tensile Tests}

The mechanical properties of the scaffolds were analysed by a single column tabletop testing system Instron 5944 at room temperature in air. The samples were cut in rectangular strips $20 \mathrm{~mm}$ wide and $40 \mathrm{~mm}$ long, and mounted with side action grip clamps with flat jaw faces. The average sample thickness was (205 \pm 4$) \mu \mathrm{m}$ for the composition 3D-ES PCL-PGS, $(240 \pm 9) \mu \mathrm{m}$ for 3D-ES PCL-PGS-5BGs and $(280 \pm 9) \mu \mathrm{m}$ for 3D-ES PCL-PGS-10BGs. The rate of extension was set at $10 \mathrm{~mm} / \mathrm{min}$. Ten samples for each scaffold composition were analysed and the data are given in the text as average \pm standard deviation. The load vs. extension graphs show the average curve within a shaded region, which indicates the values measured for all replicates. The elastic moduli of all samples were calculated within the linear region of the stress-strain curve between $0.001-0.005$ strain.

\subsubsection{In vitro Degradation Tests}

The degradation behaviour of the scaffolds was evaluated in PBS solution in static conditions at $37^{\circ} \mathrm{C}$ for 3 months. PCL-PGS scaffolds without BGs were used as control. Changes in $\mathrm{pH}$, water absorption and weight loss were recorded. Squared samples $\left(1 \mathrm{~cm}^{2}\right)$ were placed in 24-well plates with low evaporation lid in a humid atmosphere, and $2.5 \mathrm{~mL}$ of PBS was added to cover the samples completely. To determine initial dry weight, the samples were weighed with a $0.1 \mathrm{mg}$ resolution balance (Sartoruis, CP3245). The plates were then incubated at $37^{\circ} \mathrm{C}$ under humid conditions. For each timepoint, the weight loss of the samples and $\mathrm{pH}$ changes in the PBS solution were recorded (five measurements per time point). The weight loss was calculated by recording the mass of the samples before $\left(m_{i}\right)$ and after $\left(m_{f}\right)$ the degradation period: weight loss $=\left(m_{f}-m_{i}\right) / m_{i}$. The samples were then washed with deionised water and left to dry overnight, before weighing again to determine the degraded dry weight $\left(m_{d}\right)$. The percentage water absorption of each sample was calculated as $\left(m_{f}-m_{d}\right) / m_{d}$.

The mechanical properties of $2 \times 4 \mathrm{~cm}^{2}$ rectangular samples, incubated in PBS at $37^{\circ} \mathrm{C}$, were also analysed after one and two months of degradation.

\subsubsection{Biocompatibility Tests}

$3 \mathrm{~T} 3$ cells (fibroblasts) were harvested and cultured from mice. After thawing, the 3T3 cells were grown in Alpha MEM Medium (BioWhittaker Reagents, Lonza Inc, Manchester, UK) that was complemented with 10\% foetal bovine serum (Sigma Aldrich), 1\% antibiotic and antimycotic $(100 \mu \mathrm{g} / \mathrm{ml}$ penicillin, $100 \mathrm{mg} / \mathrm{ml}$ streptomycin, and $0.25 \mu \mathrm{g} / \mathrm{ml}$ amphotericin B; Sigma Aldrich) and $1 \%$ of L-Glutamine (Sigma Aldrich). Cell cultures were maintained in $75 \mathrm{~cm}^{2}$ flasks (Costar, Corning Inc., Corning, NY, USA) in standard culture conditions of $37^{\circ} \mathrm{C}$ and $5 \% \mathrm{CO}_{2}$. Cells were passaged every 2 days after harvesting them by trypsinization using 0.05\% Trypsin-EDTA solution (Gibco, Fisher Scientific, Loughborough, UK) at $80-90 \%$ culture confluence and further sub-cultivated into culture 
flasks. 3T3 cells were counted using Trypan blue (Sigma Aldrich) and a hemocytometer. The cells were seeded in 96-well flat-bottomed plates using a pipette. A total of 10,000 cells were deposited in 36 wells of a 96-well plate.

The scaffolds (with an area of $1 \mathrm{~cm}^{2}$ ) were sterilised using ethanol, following a procedure reported in the literature $[8,13,14]$. Three samples for each type of scaffold were tested. The scaffolds were covered with $2.5 \mathrm{~mL}$ of media and placed in an incubator under standard culture conditions of $37^{\circ} \mathrm{C}$ and $5 \% \mathrm{CO}_{2}$. A total of $2.5 \mathrm{~mL}$ of medium without scaffold or cells was incubated alongside the others. After 1,3 and 7 days, $0.2 \mathrm{~mL}$ of the medium covering the scaffolds was taken using a pipette and placed on top of the cells in the 96-well plates. Negative control samples (cells) were incubated in the culture medium Alpha MEM; some of them were incubated alongside the scaffolds. Positive control samples (cells) were treated with Industrial Methylated Spirit (IMS) just 20 min before testing cell viability. Following this step, the cells were incubated for $24 \mathrm{~h}$ and viability was measured using the PrestoBlue Cell Viability Reagent Protocol and assessed using fluorescence measurements. All conditions were tested in triplicate wells.

After being incubated for $24 \mathrm{~h}$, culture supernatant was slowly aspirated using a pipette linked to an aspirator. $0.1 \mathrm{ml}$ of $1 / 10$ PrestoBlue in medium solution was deposited in each well plate containing cells and in three wells without cells to measure the background fluorescence of the PrestoBlue. The well plate was covered with aluminium foil and placed in an incubator for $30 \mathrm{~min}$. Fluorescence/viability was assessed using a plate reader. During the reading, the 96-well plate was agitated for $5 \mathrm{~s}$, and measurements of fluorescence were done at $560 \mathrm{~nm}$ excitation and $590 \mathrm{~nm}$ emission. Measurements corresponding to the negative control were considered as 100\% cell viability. Viability values for the other samples were normalised to this value.

\section{Results and Discussion}

Here, as demonstrated by NMR and FTIR analyses, PGS was successfully synthesised using a two-step procedure: polycondensation of glycerol and sebacic acid, followed by thermal crosslinking [12]. The ${ }^{1} \mathrm{H}$ NMR spectrum in Figure 1a shows the methylene peaks of sebacic acid at 1.30, 1.62 and $2.35 \mathrm{ppm}$, and the peaks of glycerol between 4.05 and $4.35 \mathrm{ppm}$ [15]. The signature bands of PGS are identifiable also in the FTIR spectrum (Figure 1b): the peaks at 2927 and $2851 \mathrm{~cm}^{-1}$ are attributed to alkene $\left(-\mathrm{CH}_{2}\right)$ groups; the intense peaks at 1732 and $1164 \mathrm{~cm}^{-1}$ are due to $\mathrm{C}=\mathrm{O}$ and $\mathrm{C}-\mathrm{O}$ stretching, respectively, and confirm the formation of ester bonds [16-18].

\subsection{Fabrication of Multi-layer PCL-PGS-BGs Scaffolds}

After synthesis, PGS was blended with PCL (in a 1:1 PCL:PGS weight ratio) to produce multi-layer scaffolds consisting of a 3D-printed grid coated with a mat of electrospun fibres. Bioactive glass microspheres with an average diameter of $(60 \pm 25) \mu \mathrm{m}$ (inset of Figure 1c) were incorporated into the 3D printed layer to achieve control over mechanical properties and degradation behaviour. The amorphous nature of the BG microspheres used was confirmed by the XRD pattern in Figure 1c, showing a broad band at diffraction angles between $25^{\circ}$ and $35^{\circ}$, due to the short-range order of the silicate structure $[19,20]$. 
(a)
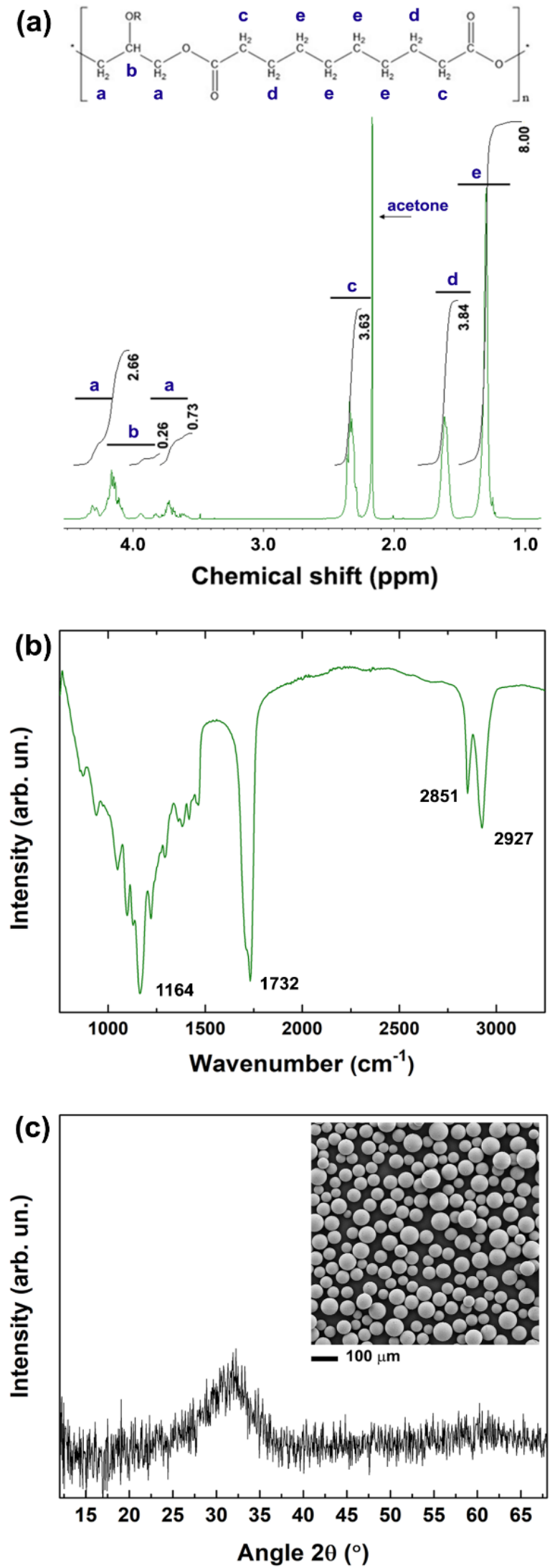

Figure 1. (a) 1H NMR spectrum of poly (glycerol sebacate) (PGS) in deuterated acetone. The typical chemical structure of PGS is shown at the top of the graph. (b) FTIR spectrum of PGS. The characteristic peaks are indicated in the graph. (c) XRD pattern of the $45 S 5$ bioactive glass microspheres and their morphology analysed by SEM (inset). 
The 3D-printed grids were characterised by interconnected pores poly-dispersed in three dimensions, due to the presence of solvent residues after layer deposition (Figure 2a,b). On one hand, the solvent facilitated the 3D printing process by reducing the viscosity of the PCL-PGS solution, promoting the dispersion of the BG microspheres, and therefore avoiding clogging the nozzle during printing. On the other hand, the low viscosity of the polymer blend determined the deformation and fusion of layers. The first layer was less affected by the deformation, while the second and the subsequent layers were squeezed and radially diffused, with the consequent formation of geometrically irregular pores. Deviations from perfect square pores have been observed in previous studies on the extrusion printing of reticular structures of soft materials, such as hydrogels and elastomers [21,22]. The fusion between adjacent PCL-PGS printed layers, due to solvent traces and low melting temperature of PGS (broad range between -20 and $40^{\circ} \mathrm{C}$ ) [16], was advantageous to create monolithic constructs with improved mechanical stability (Section 3.2). As shown in Figure $2 b$ and relative insets, the BG microspheres were predominantly uniformly distributed within the whole volume of the scaffold, even if small aggregates were also observed. The BGs were exposed on the surface of the 3D-printed scaffolds but also embedded into the polymer matrix. The exposed BG microspheres and the surface micro-porosity of the scaffolds, caused by solvent evaporation, contributed to the erosion of the systems during degradation (as discussed in Section 3.3).

One side of the 3D printed structures (the final 3D printed layer) was coated with a mat of PCL-PGS electrospun fibres. In order to achieve a strong adhesion between layers, the electrospinning process was conducted directly onto the $3 \mathrm{D}$ printed constructs. The resulting network was free from defects or beads, and formed of bundles of fibres (Figure 2c,d). the fusion between adjacent PCL-PGS fibres has been reported in the literature and attributed to the slow evaporation of the solvent [13]. The low voltage used to extrude the fibres $(8 \mathrm{kV}$ instead of $15-18 \mathrm{kV}$ reported in previous studies on 1:1 PCL:PGS solutions) [4,23] limited jet elongation and fibre stretching, likely slowing down solvent evaporation during fibre collection [24,25]. The fibres reached the collector (the 3D-printed scaffold) before complete solidification; the trapped solvent continued to diffuse out and determined coalescence at the fibre-fibre junctions and along the fibre length. Therefore, membranes consisting of interconnected fibre layers were created. The presence of solvent traces in the fibres was essential to promote adhesion between the electrospun mat and the 3D-printed structure, and ensure uniform coverage of the pores of the 3D grid (Figure 2e,f). The adhesion was tested by keeping the multi-layer PCL-PGS scaffolds in water at $37^{\circ} \mathrm{C}$ under agitation for one week. No signs of detachment of the electrospun mat from the $3 \mathrm{D}$ printed grid were observed.

In a previous study on biphasic systems formed of electrospun PCL fibres and 3D PCL/beta tri-calcium phosphate $(\beta-\mathrm{TCP})$ scaffolds, direct electrospinning onto the 3D struts was not successful in obtaining strong adhesion, due to the absence of solvent in the fibres [26]. It was instead necessary to partially melt one surface of the 3D scaffold and press it into the electrospun mat, with consequent changes in the morphology and porosity of this latter layer. In a recent work, PCL electrospun sheets have been treated with oxygen plasma, before being inserted in between cross-linked 3D-printed alginate layers [27]. The plasma treatment made the PCL fibres hydrophilic and prevented layer delamination. Our composite PCL-PGS scaffolds were instead ready to be used after electrospinning, without the need for post-processing procedures. 
(a)

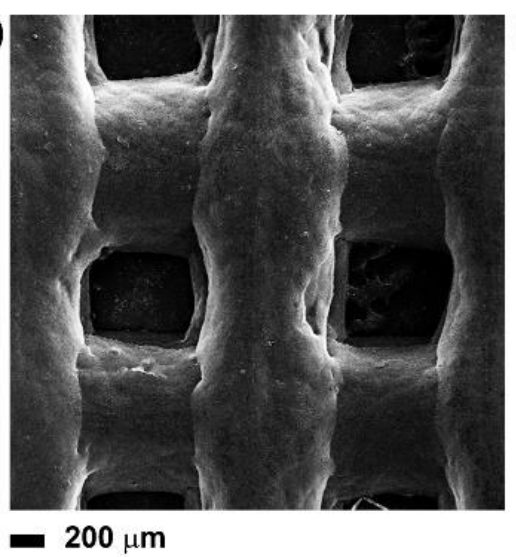

(c)

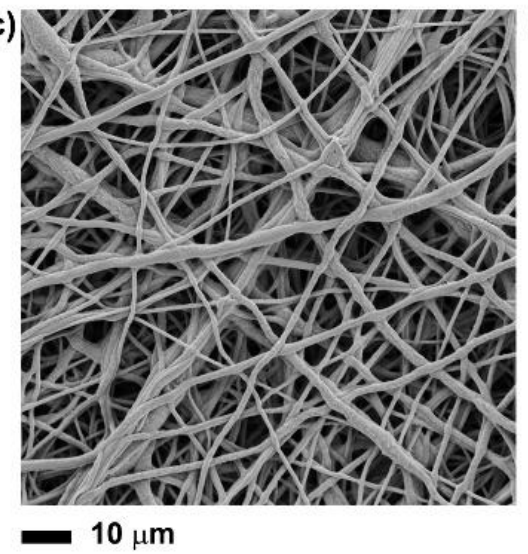

(e)

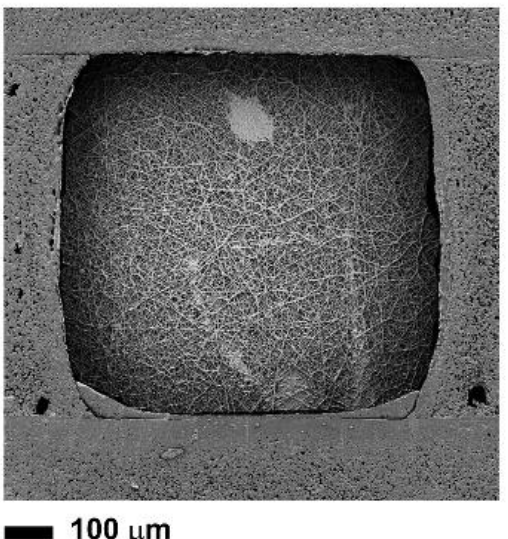

(b)

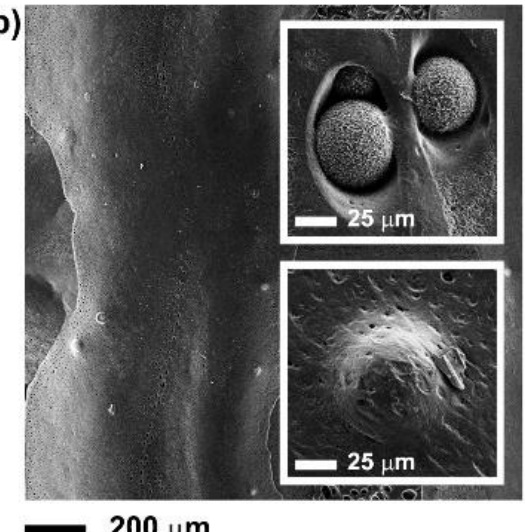

(d)

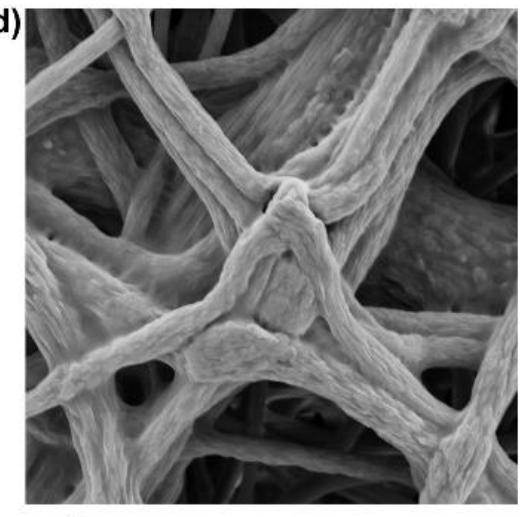

- $1 \mu \mathrm{m}$

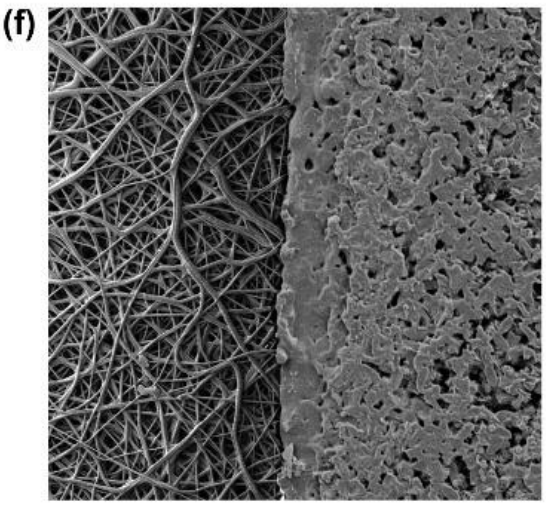

- $10 \mu \mathrm{m}$

Figure 2. SEM images at different magnifications of: (a) and (b) the 3D-printed scaffolds, showing also the BG microspheres exposed onto the surface or embedded into the polymer matrix (insets); (c) and (d) the surface of the composite scaffold covered with a layer of electrospun PCL-PGS mats, pointing out the fusion between fibres; (e) and (f) the surface of the composite scaffold without the layer of electrospun fibres.

\subsection{Characterisation of the Tensile Response of the PCL-PGS-BGs Scaffolds}

The mechanical properties of the composite scaffolds were investigated by uniaxial tensile tests. The 3D-printed PCL-PGS grids sustained an average maximum load of $4.5 \mathrm{~N}$ in tension and a maximum extension of $3.5 \mathrm{~mm}$ (Figure 3a). The addition of the electrospun layer resulted in scaffolds (referred to as 3D-ES PCL-PGS) that bore a maximum load of $6.0 \mathrm{~N}$ and extended up to $1.5 \mathrm{~mm}$ (Figure $3 \mathrm{~b}$ ). The electrospun fibres acted as a reinforcement for the assembled patches due to the excellent fibre-fibre bonding and strong interfacial adhesion between the electrospun layer and the 3D-printed one. 
Similar mechanical behaviour, even though for a different scaffold architecture, has been recorded for three-layer systems made of porous PGS tubes wrapped with PCL electrospun fibres and reinforced by PCL rings (tensile load at break of around $5.5 \mathrm{~N}$ ), where PGS significantly contributed to the elastomeric response [28]. The use of electrospun sheets to increase tensile strength in composites has been described in the literature for microfibre-based netting materials [29-31].
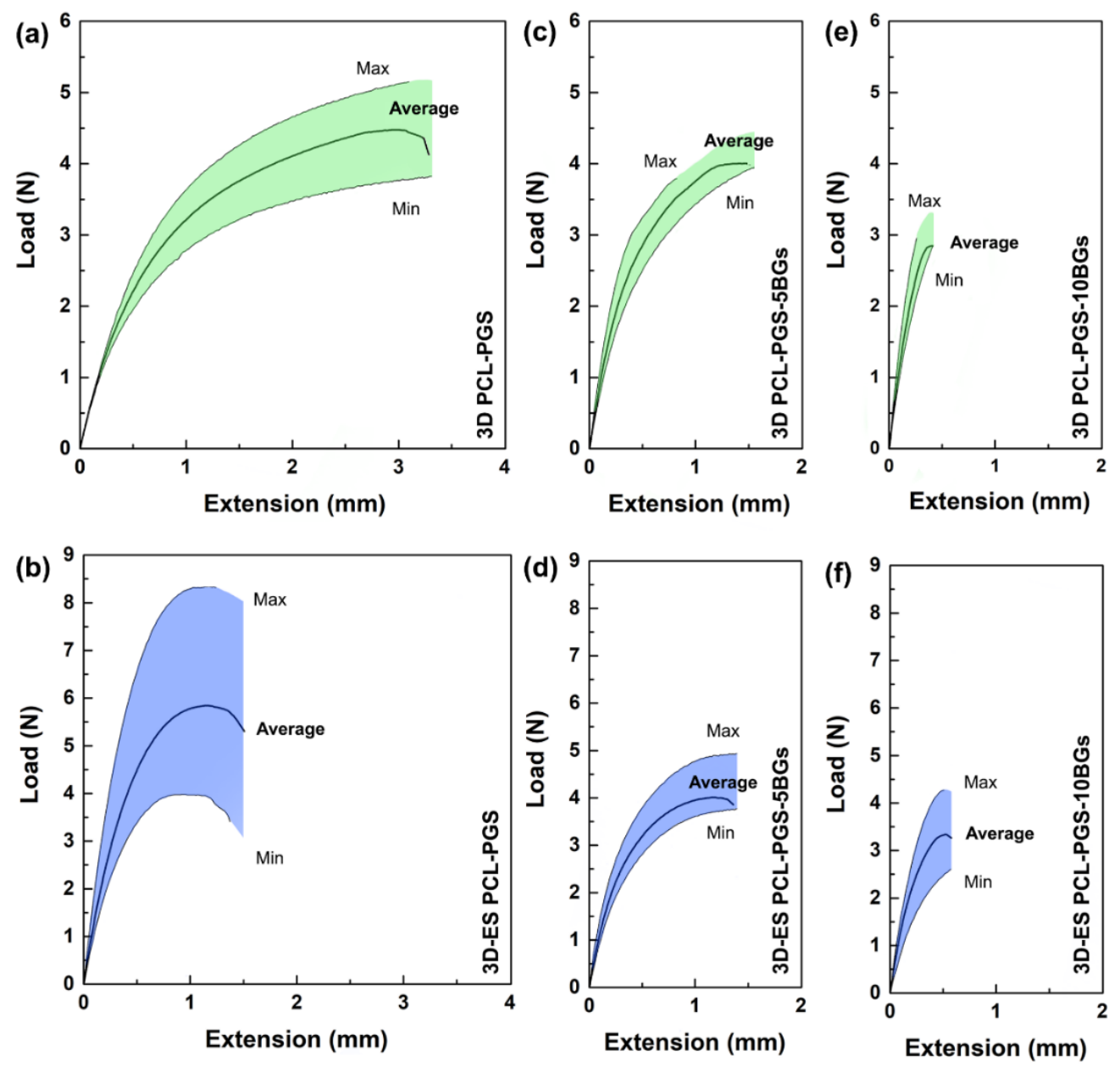

Figure 3. Load vs. extension curves of: 3D printed PCL-PGS, without (a) and with (b) the electrospun PCL-PGS mat; 3D printed PCL-PGS with $5 \mathrm{wt} \%$ of BGs, without (c) and with (d) the electrospun PCL-PGS mat; 3D printed PCL-PGS with $10 \mathrm{wt} \%$ of BGs, without (e) and with (f) the electrospun PCL-PGS mat. For each sample, the average curve is shown within a shaded region, which indicates the range of load values measured for ten replicates.

The incorporation of $5 \mathrm{wt} \%$ of bioactive glass microspheres in the PCL-PGS matrix gave a significant reduction in the sample extension before failure, with no statistically significant changes in the maximum load (Figure 3c). Similar values of load and extension at the breaking point were recorded after the deposition of the fibrous layer (Figure 3d). By increasing the BGs concentration to $10 \mathrm{wt} \%$, drops in both maximum load and extension were recorded for 3D PCL-PGS-10BGs (Figure 3e) and 3D-ES PCL-PGS-10BGs (Figure 3f).

The analysis of the Young's modulus $(E)$ better clarifies the role of the electrospun mat and BGs concentration on the mechanical properties of the scaffolds (Figure 4). The electrospun mats (ES PCL-PGS) exhibited a Young's modulus of $(66 \pm 16) \mathrm{MPa}$, which is higher than values reported in the literature for electrospun 1:1 PCL:PGS blends (in the range of 3.6-11.0 MPa) [4,13]. The cross-linking of PGS and fusion between fibres and electrospun layers are likely to be the reasons for the high values measured. It has been reported that the Young's modulus of PCL fibrous mats can be increased 
by welding procedures, such as vapour treatment and thermal annealing [32,33]. These methods induce interfibre bonding that limits fibre movement and slipping during stretching, with a consequent increase in mat resistance to deformation [34].

(a)

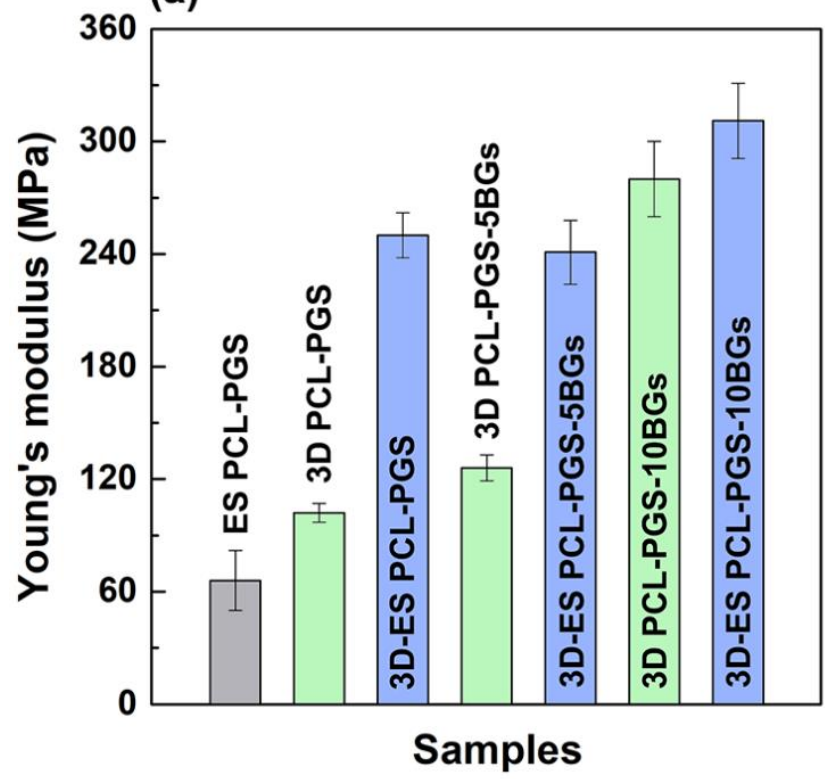

(b)

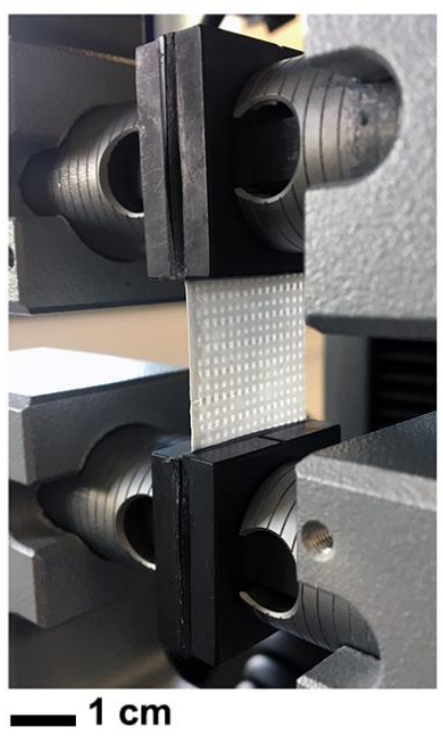

Figure 4. Values of Young's modulus for the different scaffold types (a). The photograph (b) of one composite scaffold during the tensile test shows the 3D printed grid covered by the electrospun mat.

When the fibres were deposited onto the 3D printed PCL-PGS scaffolds, a significant rise in the Young's modulus of the composite scaffolds was observed: from (102 \pm 5$) \mathrm{MPa}$ for 3D PCL-PGS samples to $(250 \pm 12) \mathrm{MPa}$ for 3D-ES PCL-PGS ones. The increase in stiffness can be associated with the excellent adhesion between the 3D-printed layer and the electrospun one, and makes the electrospun/3D-printed samples of potential interest as engineered scaffolds for stiff human tissues, such as tendons and ligaments. The effect of the electrospun fibres on the mechanical properties of the scaffolds was evident also for samples containing BG microspheres. 3D-printed scaffolds with 5 and $10 \mathrm{wt} \%$ of BGs had $E$ values of $(126 \pm 7) \mathrm{MPa}$ and $(280 \pm 20) \mathrm{MPa}$, respectively; the deposition of the electrospun mats determined an increase in Young's modulus up to $(241 \pm 17)$ MPa for 3D-ES PCL-PGS-5BGs and $(311 \pm 20)$ MPa for 3D-ES PCL-PGS-10BGs. A comparison between composite 3D-ES samples shows that $5 \mathrm{wt} \%$ of BGs had no significant impact on the stiffness, differently from $10 \mathrm{wt} \%$ BGs that induced a $30 \%$ increase in the Young's modulus. Similar values of elastic modulus in tension have been reported for polymer-BGs scaffolds for bone tissue engineering, including: $60 \mu \mathrm{m}$-thick PCL films containing $50 \mathrm{wt} \%$ of $45 \mathrm{~S} 5$ bio-glasses ( $200 \mathrm{MPa}$ ) [35], electrospun mats ( $80 \mu \mathrm{m}$ thick) of polyvinyl alcohol and $45 \mathrm{S5}$ BGs ( 250 MPa) [36], and polydimethylsiloxane-BGs-PCL monoliths (230-330 MPa) [37].

\subsection{Analysis of the Degradation Behaviour of the PCL-PGS-BGs Scaffolds}

The degradation of the composite scaffolds was studied in PBS at $37^{\circ} \mathrm{C}$ and $\mathrm{pH} 7.4$ to reproduce normal physiological conditions. Weight loss of the samples (Figure 5a) and pH changes in the buffer solution (Figure $5 b$ ) were recorded over a period of 3 months. The scaffolds without BGs and with $5 \mathrm{wt} \%$ BGs showed a similar degradation profile with a weight loss of $6.5 \%$ (3D-ES PCL-PGS) and 7.0\% (3D-ES PCL-PGS-5BGs) after 21 days; while, in the same time frame, the 3D-ES PCL-PGS-10BGs samples exhibited a $9.8 \%$ weight loss. The degradation was initiated by the hydrolysis of PGS and dissolution of the BGs microparticles [38-42]. The release of unreacted carboxylic acid groups of PGS and those formed by the cleavage of PGS ester linkages caused acidification of the PBS medium [13,23]: $\mathrm{pH} 6.0$ after 21 days. The release of alkaline ions from the scaffolds with the lowest concentration of 
BGs (5 wt\%) had a limited effect on balancing out the pH drop (pH 6.0); while $10 \mathrm{wt} \%$ BGs maintained $\mathrm{pH}$ to values higher than 6.5 for the first 14 days. The weight loss continued to increase over time for all scaffolds, reaching $10.7 \%$ for 3D-ES PCL-PGS, $15.4 \%$ for 3D-ES PCL-PGS-5BGs and $14.1 \%$ for 3D-ES PCL-PGS-10BGs, after 56 days of incubation in PBS. This corresponded to a decrease in the $\mathrm{pH}$ of the PBS medium to 5.3-5.4 for 3D-ES PCL-PGS and 3D-ES PCL-PGS-5BGs, and 6.0 for 3D-ES PCL-PGS-10BGs.
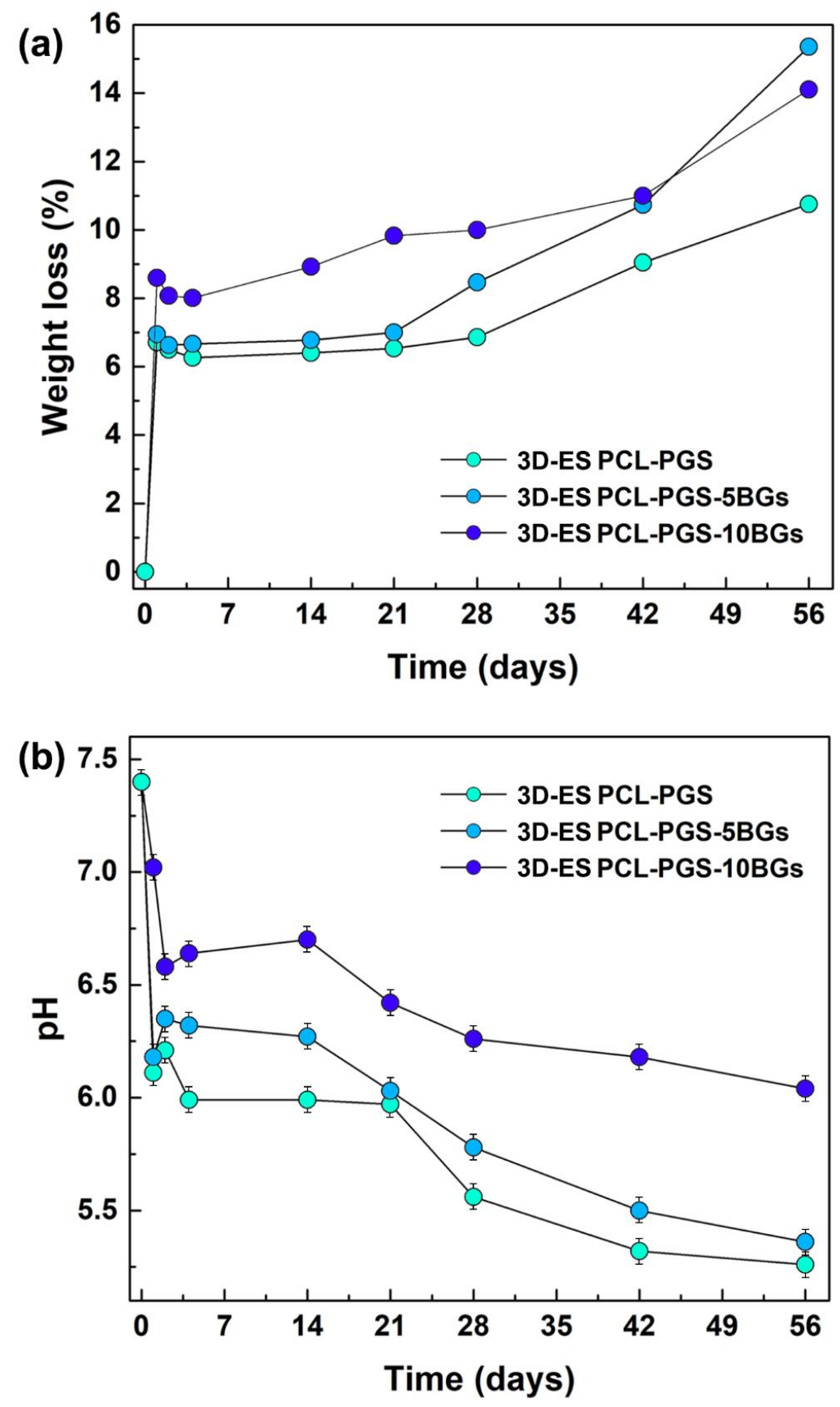

Figure 5. In vitro degradation of the composite scaffolds with and without bioactive glass microparticles: (a) percentage of weight loss and (b) $\mathrm{pH}$ changes at different timepoints. The data are represented as average \pm standard deviation for five repeats (the size of the symbol includes the error bar).

The weight loss experienced by scaffolds containing BGs indicates that both PGS and bioactive glass microparticles were released, as was also confirmed by the $\mathrm{pH}$ changes in the PBS medium, particularly for samples with $10 \mathrm{wt} \%$ BGs. As a consequence, a deterioration in the mechanical properties and 
morphology of the scaffolds was observed. As shown in Figure 6a, a significant decrease in the Young's modulus was recorded for all scaffold types after one and two months of degradation. Samples with $10 \mathrm{wt} \%$ of BGs exhibited the lowest value of Young's modulus, (36 \pm 15$) \mathrm{MPa}$, corresponding to a more than 8-fold decrease compared to the non-degraded scaffolds. SEM investigations of the electrospun layer revealed that non-fibrous regions (thin polymer films) started to emerge in between fibres and the roughness of the fibres surface increased (Figure 6b), likely due to the erosion of PGS. Pores and defects were instead detected on the surface (Figure 6c) and within the cross-section (Figure 6d,e) of the 3D-printed layer, caused by PGS and BGs release, which are ultimately responsible for the failure of the scaffolds under tension.
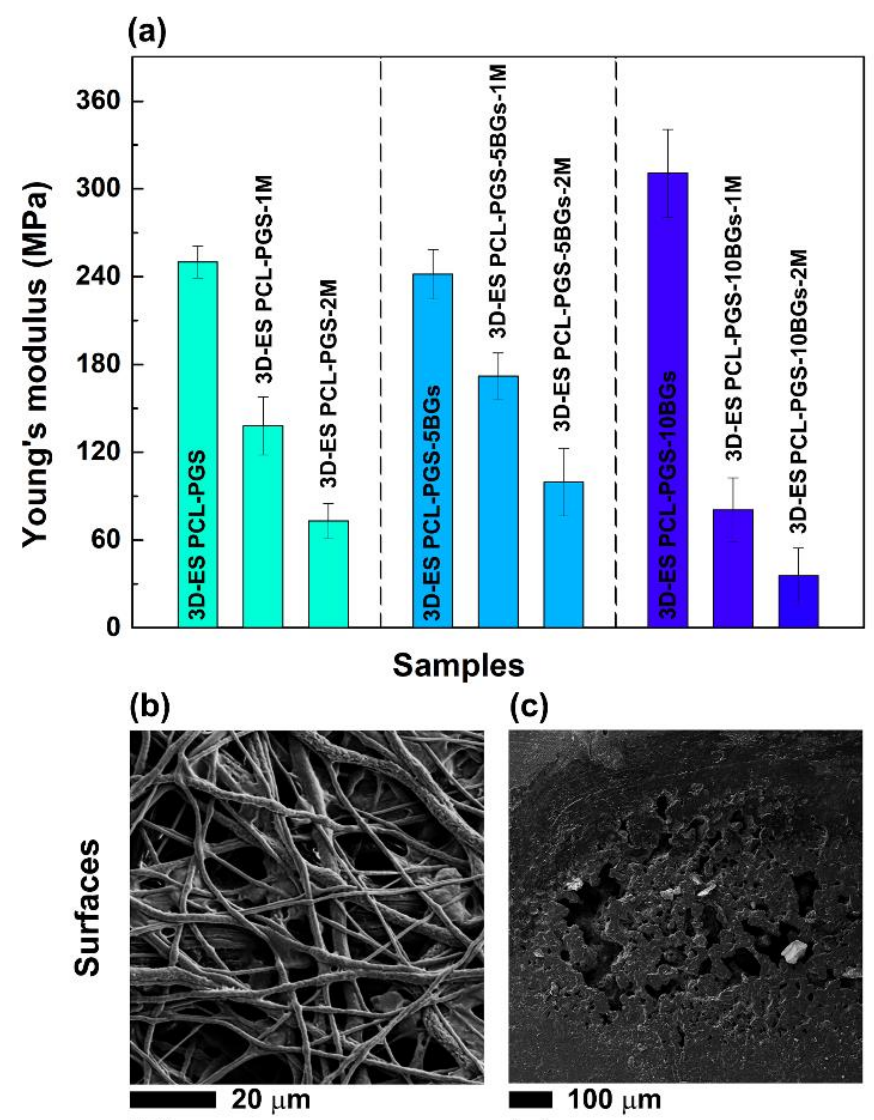

(c)

(d)

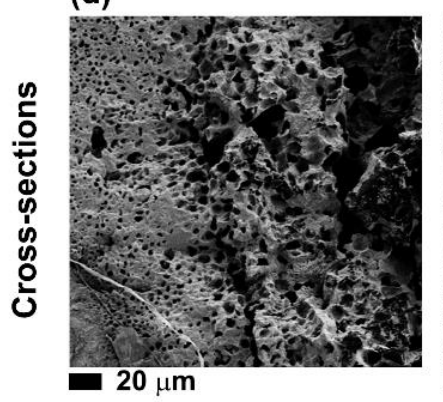

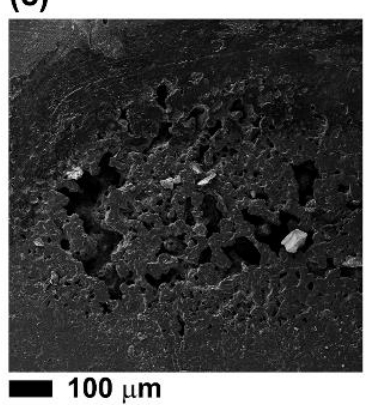

(e)

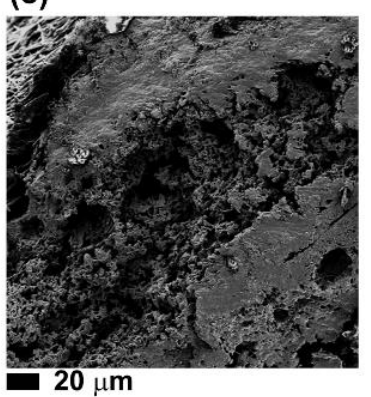

Figure 6. (a) Values of Young's modulus of the composite scaffolds after one month (3D-ES PCL-PGS-1M, 3D-ES PCL-PGS-5BGs-1M, 3D-ES PCL-PGS-10BGs-1M) and two months (3D-ES PCL-PGS-2M, 3D-ES PCL-PGS-5BGs-2M, 3D-ES PCL-PGS-10BGs-2M) of incubation in PBS at $37^{\circ} \mathrm{C}$. SEM images, after 2 months degradation, of the surface (b) of the electrospun layer and (c) 3D-printed one; (d) and (e) the cross-section of the 3D-printed layer. 


\subsection{Biocompatibility Tests}

The viability of $3 \mathrm{~T} 3$ cells exposed to the PCL-PGS extracts is shown in Figure 7. All three material compositions exhibited cell viability results above $80 \%$ for all timepoints, indicating satisfactory biocompatibility. High survival rates (higher than the control sample) were recorded for all scaffolds: 3D-ES PCL-PGS (124 $\pm 4 \%)$, 3D-ES PCL-PGS-5BGs (126 \pm 7\%) and 3D-ES PCL-PGS-10BGs $(129 \pm 4 \%)$ at day 1 . The highest viability was measured at day 2 with values of $125 \% \pm 11 \%, 137 \% \pm 11 \%$ and $134 \% \pm 6 \%$ for 3D-ES PCL-PGS, 3D-ES PCL-PGS-5BGs and 3D-ES PCL-PGS-10BGs, respectively. A decrease in cell survival was observed for all scaffold types at day 7 , particularly for 3D-ES PCL-PGS-5BGs $(84 \% \pm 9 \%)$. The results of the biocompatibility tests can be linked to the degradation of PGS, the release profile of BGs and corresponding pH changes (as shown in Figure 5). Although PGS degradation could lead to an acidic environment deleterious for cells, the release of the alkaline BGs helped to counteract the low $\mathrm{pH}$ and ensured a suitable environment for cell proliferation. Viability results demonstrated the positive effect of the addition of BGs in the 3D constructs since most cell survival rates were above that of the reference sample without BGs (3D-ES PCL-PGS).

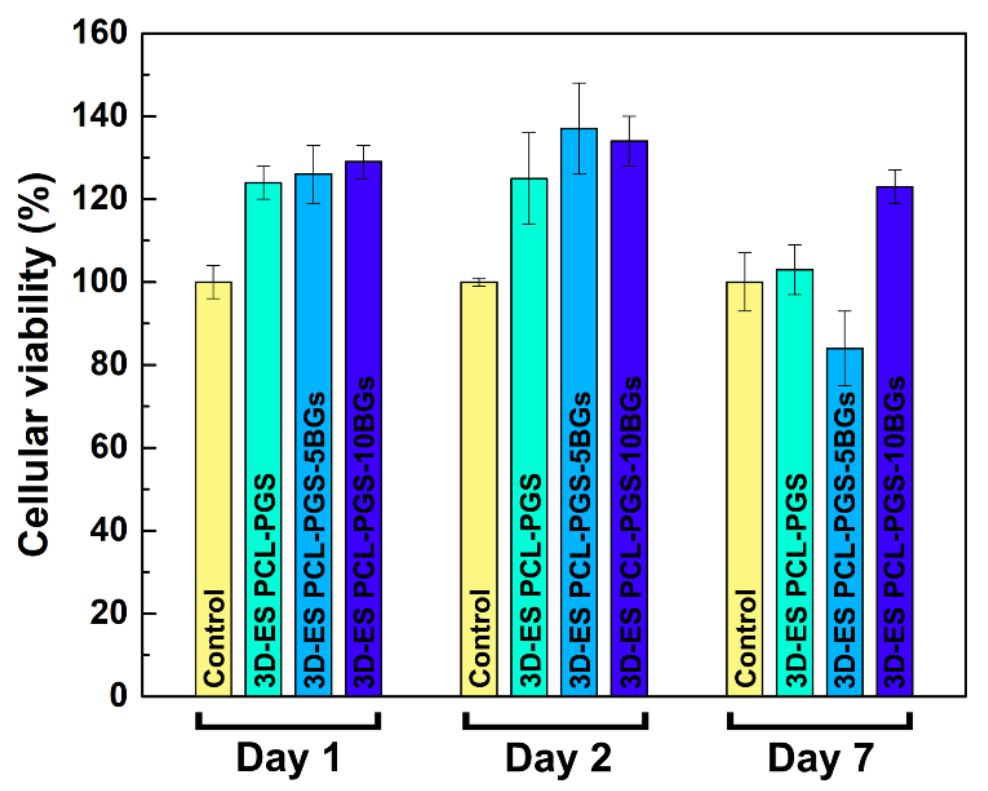

Figure 7. Percentage of 3 T3 cell viability for 3D-ES PCL-PGS scaffolds without and with bioactive glasses for three times points (1, 2 and 7 days).

\section{Conclusions}

Through this work, the design, fabrication and characterisation of scaffolds meeting various requirements for tissue engineering have been demonstrated. Electrospinning and 3D printing have been combined to manufacture biocompatible patches of PCL-PGS blends containing bioactive glasses. The scaffolds exhibited a multiscale three-dimensional porosity that is known to be beneficial for cell growth and infiltration, and the transport of nutrients and metabolic waste (not demonstrated in this work). In addition, the use of PGS and bioactive glasses allowed control over degradation and mechanical properties. The strategy used for the fabrication of the hybrid scaffolds has proved effective in achieving excellent adhesion between the 3D-printed layer, which provided mechanical support to the whole structure, and the electrospun mat, which acted as a biomimetic layer. The presence of the PCL-PGS electrospun network enhanced the stiffness of the scaffolds and gave a 2.5-fold increase of the Young's modulus. The addition of the bioactive glasses, although it affected the mechanical response of the scaffolds only slightly (a 30\% increase in elastic modulus for samples with $10 \mathrm{wt} \%$ BGs), impacted the material degradation in vitro and balanced the acidic character of PGS. After 3 months of in vitro degradation tests, the $\mathrm{pH}$ of the PBS medium, where the scaffolds were incubated, dropped to 5.3 (from 
7.4) when no BGs were used; while it only reached values of 6.0 for scaffolds containing $10 \mathrm{wt} \%$ BGs. This had a positive effect on the growth of fibroblasts, whose viability was sustained longer in vitro for scaffolds with $10 \mathrm{wt} \%$ BGs. The composite scaffolds can find potential applications in tendon and ligament tissue engineering, since they meet the mechanical requirements of native tissues (elastic modulus in the range of 100-300 MPa) [43], in agreement with previous studies [44]. For these specific tissue engineering applications, scaffolds with anisotropic characteristics can be manufactured using the fabrication method discussed in this work.

Author Contributions: A.B.R.T. carried out the experiment. All three authors participated in the writing of the manuscript. E.M. and J.K.C. supervised the project. All authors have read and agreed to the published version of the manuscript.

Funding: The authors thank the LoughboroughUniversity for funding the PhD of Adja B. R. Touré.

Acknowledgments: Adja B. R. Touré thanks the School of Aeronautical, Automotive, Chemical and Materials Engineering of Loughborough University for funding the PhD scholarship.

Conflicts of Interest: The authors declare no conflict of interest.

\section{References}

1. Jammalamadaka, U.; Tappa, K. Recent advances in biomaterials for 3D printing and tissue engineering. J. Funct. Biomater. 2018, 9, 22. [CrossRef] [PubMed]

2. O'Brien, F.J. Biomaterials \& scaffolds for tissue engineering. Mater. Today 2011, 14, 88-95.

3. Chen, F.-M.; Liu, X. Advancing biomaterials of human origin for tissue engineering. Prog. Polym. Sci. 2016, 53, 86-168. [CrossRef] [PubMed]

4. Masoumi, N.; Larson, B.J.; Annabi, N.; Kharaziha, M.; Zamanian, B.; Shapero, K.S.; Cubberley, A.T.; Camci-Unal, G.; Manning, K.B.; Mayer, J.E., Jr.; et al. Electrospun PGS: PCL microfibers align human valvular interstitial cells and provide tunable scaffold anisotropy. Adv. Healthc. Mater. 2014, 3, 929-939. [CrossRef] [PubMed]

5. Sant, S.; Iyer, D.; Gaharwar, A.K.; Patel, A.; Khademhosseini, A. Effect of biodegradation and de novo matrix synthesis on the mechanical properties of valvular interstitial cell-seeded polyglycerol sebacate-polycaprolactone scaffolds. Acta Biomater. 2013, 9, 5963-5973. [CrossRef]

6. Masoumi, N.; Annabi, N.; Assmann, A.; Larson, B.L.; Hjortnaes, J.; Alemdar, N.; Kharaziha, M.; Manning, K.B.; Mayer, J.E., Jr.; Khademhosseini, A. Tri-layered elastomeric scaffolds for engineering heart valve leaflets. Biomaterials 2014, 35, 7774-7785. [CrossRef]

7. Rai, R.; Tallawi, M.; Frati, C.; Falco, A.; Gervasi, A.; Quaini, F.; Roether, J.A.; Hochburger, T.; Schubert, D.W.; Seik, L.; et al. Bioactive electrospun fibers of poly(glycerol sebacate) and poly(E-caprolactone) for cardiac patch application. Adv. Healthc. Mater. 2015, 4, 2012-2025. [CrossRef]

8. Tallawi, M.; Dippold, D.; Rai, R.; D’Atri, D.; Roether, J.A.; Schubert, D.W.; Rosellini, E.; Engel, F.B.; Boccaccini, A.R. Novel PGS/PCL electrospun fiber mats with patterned topographical features for cardiac patch applications. Mater. Sci. Eng. C 2016, 69, 569-576. [CrossRef]

9. Yang, Y.; Lei, D.; Huang, S.; Yang, Q.; Song, B.; Guo, Y.; Shen, A.; Yuan, Z.; Li, S.; Qing, F.-L.; et al. Elastic 3D-printed hybrid polymeric scaffold improves cardiac remodeling after myocardial infarction. Adv. Healthc. Mater. 2019, 8, 1900065. [CrossRef]

10. Salehi, S.; Czugala, M.; Stafiej, P.; Fathi, M.; Bahners, T.; Gutmann, J.S.; Singer, B.B.; Fuchsluger, T.A. Poly (glycerol sebacate)-poly (e-caprolactone) blend nanofibrous scaffold as intrinsic bio- and immunocompatible system for corneal repair. Acta Biomater. 2017, 50, 370-380. [CrossRef]

11. Giannitelli, S.M.; Mozetic, P.; Trombetta, M.; Rainer, A. Combined additive manufacturing approaches in tissue engineering. Acta Biomater. 2015, 24, 1-11. [CrossRef] [PubMed]

12. Wang, Y.; Ameer, G.A.; Sheppard, B.J.; Langer, R. A tough biodegradable elastomer. Nat. Biotechnol. 2002, 20, 602-606. [CrossRef] [PubMed]

13. Vogt, L.; Rivera, L.R.; Liverani, L.; Piegat, A.; El Fray, M.; Boccaccini, A.R. Poly(e-caprolactone)/poly(glycerol sebacate) electrospun scaffolds for cardiac tissue engineering using benign solvents. Mater. Sci. Eng. C 2019, 103, 109712. [CrossRef] [PubMed] 
14. Liverani, L.; Piegat, A.; Niemczyk, A.; El Fray, M.; Boccaccini, A.R. Electrospun fibers of poly (butylene succinate-co-dilinoleic succinate) and its blend with poly (glycerol sebacate) for soft tissue engineering applications. Eur. Polym. J. 2016, 81, 295-306. [CrossRef]

15. Patel, A.; Gaharwar, A.K.; Iviglia, G.; Zhang, H.; Mukundan, S.; Mihaila, S.M.; Demarchi, D.; Khademhosseini, A. Highly elastomeric poly(glycerol sebacate)-co-poly(ethylene glycol)amphiphilic block copolymers. Biomaterials 2013, 34, 3970-3983. [CrossRef]

16. Rai, R.; Tallawi, M.; Grigore, A.; Boccaccini, A.R. Synthesis, properties and biomedical applications of poly(glycerol sebacate) (PGS): A review. Prog. Polym. Sci. 2012, 37, 1051-1078. [CrossRef]

17. Aydin, H.M.; Salimi, K.; Rzayevc, Z.M.O.; Pişkin, E. Microwave-assisted rapid synthesis of poly(glycerol-sebacate) elastomers. Biomater. Sci. 2013, 1, 503-509. [CrossRef]

18. Jia, Y.; Wang, W.; Zhou, X.; Nie, W.; Chen, L.; He, C. Synthesis and characterization of poly(glycerol sebacate)-based elastomeric copolyesters for tissue engineering applications. Polym. Chem. 2016, 7, 2553-2564. [CrossRef]

19. Zarifah, N.A.; Lim, W.F.; Matori, K.A.; Sidek, H.A.A.; Wahab, Z.A.; Zainuddin, N.; Salleh, M.A.; Fadilah, B.N.; Fauzan, A.N. An elucidating study on physical and structural properties of 4555 glass at different sintering temperatures. J. Non-Cryst. Solids 2015, 412, 24-29. [CrossRef]

20. Chen, Q.Z.; Rezwan, K.; Armitage, D.; Nazhat, S.N.; Boccaccini, A.R. The surface functionalization of $45 S 5$ Bioglass-based glass-ceramic scaffolds and its impact on bioactivity. J. Mater. Sci. Mater. Med. 2006, 17, 979-987. [CrossRef]

21. He, Y.; Yang, F.F.; Zhao, H.M.; Gao, Q.; Xia, B.; Fu, J.Z. Research on the printability of hydrogels in 3D bioprinting. Sci. Rep. 2016, 6, 29977. [CrossRef] [PubMed]

22. Ozbolat, V.; Dey, M.; Ayan, B.; Povilianskas, A.; Demirel, M.C.; Ozbolat, I.T. 3D printing of PDMS improves its mechanical and cell adhesion properties. ACS Biomater. Sci. Eng. 2018, 4, 682-693. [CrossRef]

23. Salehi, S.; Fathi, M.; Javanmard, S.H.; Bahners, T.; Gutmann, J.S.; Ergun, S.; Steuhl, K.P.; Fuchsluger, T.A. Generation of PGS/PCL blend nanofibrous scaffolds mimicking corneal stroma structure. Macromol. Mater. Eng. 2014, 299, 455-469. [CrossRef]

24. Thompson, C.J.; Chase, G.G.; Yarin, A.L.; Reneker, D.H. Effects of parameters on nanofiber diameter determined from electrospinning model. Polymer 2017, 48, 6913-6922. [CrossRef]

25. Wang, P.; Mele, E. Effect of antibacterial plant extracts on the morphology of electrospun poly (lactic acid) fibres. Materials 2018, 11, 923. [CrossRef]

26. Vaquette, C.; Fan, W.; Xiao, Y.; Hamlet, S.; Hutmacher, D.W.; Ivanovski, S. A biphasic scaffold design combined with cell sheet technology for simultaneous regeneration of alveolar bone/periodontal ligament complex. Biomaterials 2012, 33, 5560-5573. [CrossRef]

27. Yoon, Y.; Kim, C.H.; Lee, J.E.; Yoon, J.; Lee, N.K.; Kim, T.H.; Park, S.-H. 3D bioprinted complex constructs reinforced by hybrid multilayers of electrospun nanofiber sheets. Biofabrication 2019, 11, 025015. [CrossRef]

28. Wu, W.; Jia, S.; Chen, W.; Liu, X.; Zhang, S. Fast degrading elastomer stented fascia remodels into tough and vascularized construct for tracheal regeneration. Mater. Sci. Eng. C 2019, 101, 1-14. [CrossRef]

29. Hakimi, O.; Mouthuy, P.A.; Zargar, N.; Lostis, E.; Morrey, M.; Carr, A. A layered electrospun and woven surgical scaffold to enhance endogenous tendon repair. Acta Biomater. 2015, 26, 124-135. [CrossRef]

30. Kim, S.J.; Jang, D.H.; Park, W.H.; Min, B.-M. Fabrication and characterization of 3-dimensional PLGA nanofiber/microfiber composite scaffolds. Polymer 2010, 51, 1320-1327. [CrossRef]

31. Rajzer, I. Fabrication of bioactive polycaprolactone/hydroxyapatite scaffolds with final bilayer nano-/micro-fibrous structures for tissue engineering application. J. Mater. Sci. 2014, 49, 5799-5807. [CrossRef]

32. Li, H.; Zhu, C.; Xue, J.; Ke, Q.; Xia, Y. Enhancing the mechanical properties of electrospun nanofiber mats through controllable welding at the cross points. Macromol. Rapid Commun. 2017, 38, 1600723. [CrossRef] [PubMed]

33. Wu, T.; Li, H.; Xue, J.; Mo, X.; Xia, Y. Photothermal welding, melting, and patterned expansion of nonwoven mats of polymer nanofibers for biomedical and printing applications. Angew. Chem. 2019, 131, 16568-16573. [CrossRef]

34. Balzamo, G.; Zhang, X.; Bosbach, W.A.; Mele, E. In-situ formation of polyvinylidene fluoride microspheres within polycaprolactone electrospun mats. Polymer 2020, 186, 122087. [CrossRef] 
35. Mohammadkhah, A.; Marquardt, L.M.; Sakiyama-Elbert, S.E.; Day, D.E.; Harkins, A.B. Fabrication and characterization of poly-( $\varepsilon$ )-caprolactone and bioactive glass composites for tissue engineering applications. Mater. Sci. Eng. C 2015, 49, 632-639. [CrossRef]

36. Shankhwar, N.; Kumar, M.; Mandal, B.B.; Srinivasan, A. Novel polyvinyl alcohol-bioglass 45 S5 based composite nanofibrous membranes as bone scaffolds. Mater. Sci. Eng. C 2016, 69, 1167-1174. [CrossRef]

37. Chen, J.; Du, Y.; Que, W.; Xing, Y.; Lei, B. Content-dependent biomineralization activity and mechanical properties based on polydimethylsiloxane-bioactive glass-poly(caprolactone) hybrids monoliths for bone tissue regeneration. RSC Adv. 2015, 5, 61309-61317. [CrossRef]

38. Maquet, V.; Boccaccini, A.R.; Pravata, L.; Notingher, I.; Jérôme, R. Preparation, characterization, and in vitro degradation of bioresorbable and bioactive composites based on Bioglass-Filled polylactide foams. J. Biomed. Mater. Res. Part A 2003, 66, 335-346. [CrossRef]

39. Liu, X.; Rahaman, M.N.; Day, D.E. Conversion of melt-derived microfibrous borate (13-93B3) and silicate (45S5) bioactive glass in a simulated body fluid. J. Mater. Sci. Mater. Med. 2013, 24, 583-595. [CrossRef]

40. Maquet, V.; Boccaccini, A.R.; Pravata, L.; Notingher, I.; Jérôme, R. Porous poly(a-hydroxyacid)/Bioglass composite scaffolds for bone tissue engineering. I: Preparation and in vitro characterisation. Biomaterials 2004, 25, 4185-4194. [CrossRef]

41. Jones, J.R. Review of bioactive glass: From Hench to hybrids. Acta Biomater. 2013, 9, 4457-4486. [CrossRef] [PubMed]

42. Fernandes, J.S.; Gentile, P.; Martins, M.; Neves, N.M.; Miller, C.; Crawford, A.; Pires, R.A.; Hatton, P.; Reis, R.L. Reinforcement of poly-l-lactic acid electrospun membranes with strontium borosilicate bioactive glasses for bone tissue engineering. Acta Biomater. 2016, 44, 168-177. [CrossRef] [PubMed]

43. Pauly, H.M.; Kelly, D.J.; Popat, K.C.; Trujillo, N.A.; Dunne, N.J.; McCarthy, H.O.; Donahue, T.L.H. Mechanical properties and cellular response of novel electrospun nanofibers for ligament tissue engineering: Effects of orientation and geometry. J. Mech. Behav. Biomed. Mater. 2016, 61, 258-270. [CrossRef] [PubMed]

44. Lim, W.L.; Liau, L.L.; Ng, M.H.; Chowdhury, S.R.; Law, J.X. Current progress in tendon and ligament tissue engineering. Tissue Eng. Regen. Med. 2019, 16, 549-571. [CrossRef]

(C) 2020 by the authors. Licensee MDPI, Basel, Switzerland. This article is an open access article distributed under the terms and conditions of the Creative Commons Attribution (CC BY) license (http://creativecommons.org/licenses/by/4.0/). 